\title{
ARTICLE
}

\section{A novel deleterious mutation in the COMP gene that causes pseudoachondroplasia}

Huaichao Luo ${ }^{1,2,7}$, Sisi Yu ${ }^{3,7}$, Ying Lin ${ }^{1}$, Qi Guo ${ }^{4}$, Rongchuan $\mathrm{Ma}^{5}$, Zimeng $\mathrm{Ye}^{1}$, Yanan $\mathrm{Di}^{1}$, Ning Li ${ }^{1}$, Yuanying Miao ${ }^{1}$, Yu Zhou ${ }^{1}$, Yuanfeng $\mathrm{Li}^{1}$, Jiyun Yang ${ }^{1,7}$ and Zhenglin Yang ${ }^{1,6,7}$

Pseudoachondroplasia (PSACH) is a rare and severe genetic disease; therefore, an accurate molecular diagnosis is essential for appropriate disease treatment and family planning. Currently, the diagnosis of PSACH is based mainly on family history, physical examination and radiographic evaluation. Genetic studies of patients with PSACH in Chinese populations have been very limited. With the application of next-generation sequencing (NGS), a comprehensive molecular diagnosis of PSACH is now possible. The purpose of this study was to perform comprehensive NGS-based molecular diagnoses for patients with PSACH in China. We investigated the molecular genetics of one suspected PSACH family in this study. The DNA sample from the proband was sequenced using a custom capture panel that included 249 bone disease genes. Variant calls were filtered and annotated using an in-house automated pipeline. Then, we confirmed the variants by Sanger sequencing in three family members. After co-segregation analysis, the variant, c.1160_1162del of the COMP gene, was identified as a novel mutation responsible for this spontaneous form of PSACH.

Human Genome Variation (2016) 3, 16009; doi:10.1038/hgv.2016.9; published online 9 June 2016

\section{INTRODUCTION}

Pseudoachondroplasia (PSACH; OMIM\# 177170 is a rare disorder with an estimated birth prevalence of 1/30,000 (www.orpha.net); however, the accurate birth prevalence is not known because newborns with PSACH are indistinguishable from normal babies at birth, without genetic testing. Patients with PSACH share common clinical characteristics such as disproportionate short stature, brachydactyly, lower limb anomalies, loose joints, ligamentous laxity and early-onset osteoarthritis. ${ }^{1}$ This disorder is not diagnosed until $\sim 2$ years of age, with the onset of a waddling gait, lax joints and diminished linear growth. ${ }^{2}$ These PSACH clinical manifestations are very similar to those observed for other bone dysplasias such as achondroplasia (OMIM\# 100800) and hypochondroplasia (OMIM\# 146000). Consequently, it is very difficult to accurately diagnose individuals with PSACH based solely on physical examination and radiographic evaluation.

Currently, the diagnosis of PSACH is based mainly on family history, physical examination and radiographic evaluation, and genetic studies of patients with PSACH in Chinese populations are limited. For comprehensive diagnostics of early-stage PSACH, reproductive decision-making and genetic counseling purposes, an accurate molecular diagnosis is essential. Until recently, most laboratories have conducted Sanger sequencing to detect the COMP mutations of suspected patients when faced with clinical diagnostic uncertainty. Previous publications have suggested that PSACH results exclusively from COMP mutations. ${ }^{1}$ However, this method is costly and time consuming. Moreover, this method is difficult for high-throughput diagnosis and carrier screening. With the application of next-generation sequencing (NGS), a promising alternative for the comprehensive molecular diagnosis of PSACH is now possible. Coupled with DNA enrichment technology, NGS provides rapid and cost-effective parallel sequencing of a large panel of disease genes to diagnose suspected PSACH patients.

In this study we studied a Chinese family diagnosed with suspected PSACH based on clinical and radiologic findings. The DNA sample from the proband was sequenced using a custom capture panel that included 249 bone disease genes to detect potential disease-causing variants. This study represents the first report of an NGS-based, comprehensive molecular diagnostic test in patients with PSACH and demonstrates that NGS is an accurate and rapid method for the genetic diagnosis of suspected cases of PSACH.

\section{MATERIALS AND METHODS}

\section{Subjects}

Approval was obtained from the Institutional Review Boards of the Sichuan Academy of Medical Sciences and Sichuan Provincial People's Hospital. Written informed consent was obtained from all subjects before inclusion in the study. The study was conducted in accordance with the tenets of the Declaration of Helsinki and its subsequent revisions. The proband was initially diagnosed with mucopolysaccharidosis, which was subsequently ruled out using enzyme activity analysis. The family then contacted the genetic clinic of the Sichuan Academy of Medical Sciences and Sichuan Provincial People's Hospital (Figure 1). The study participants included the proband (II-1) and unaffected parents (I-1 and I-2), who received comprehensive physical examinations; their blood samples were collected

\footnotetext{
${ }^{1}$ Sichuan Provincial Key Laboratory for Human Disease Gene Study, The Institute of Laboratory Medicine, Hospital of University of Electronic Science and Technology of China and Sichuan Provincial People's Hospital, Sichuan, China; ${ }^{2}$ Clinical Laboratory Department, Sichuan Cancer Hospital and Institute, Sichuan, China; ${ }^{3}$ Department of Medical Oncology,

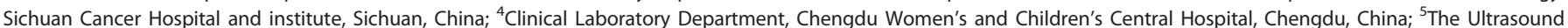
Department, Chengdu Women's and Children's Hospital, Sichuan, China and ${ }^{6}$ Clinical Medicine College, Sichuan Medical University, Sichuan, China.

Correspondence: J Yang (yangjiyun@yeah.net) or Z Yang (zliny@yahoo.com)

${ }^{7}$ These authors contributed equally to this work.

Received 11 September 2015; revised 25 November 2015; accepted 27 March 2016
} 
for further genetic study. The proband additionally received comprehensive radiographic evaluation.

\section{Illumina library preparation}

Genomic DNA of the proband was extracted from whole blood using a DNA Extraction kit (TIANGEN, Beijing, China) according to the manufacturer's instructions. The DNA was quantified using a Nanodrop 2000 (Thermo Fisher Scientific, Waltham, MA, USA). Genomic DNA (3 $\mu \mathrm{g})$ was fragmented by nebulization, and the fragmented DNA was endrepaired and A-tailed using standard protocols. Illumina adapters were ligated to the A-tailed fragments, and the products were size-selected for a 350 - to 400-base pair product. The size-selected product was PCR

\section{I}

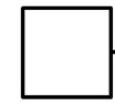

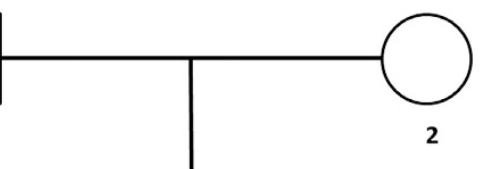

II

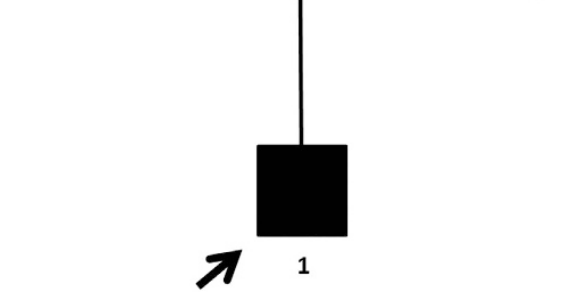

Figure 1. Pedigree of participating subjects in the study of the suspected PSACH family. Solid symbol indicates affected individuals, open symbols indicate unaffected individuals and the arrow indicates the proband. PSACH, pseudoachondroplasia. amplified, and the final product was validated using the Agilent Bioanalyzer (Agilent Technologies, Santa Clara, CA, USA).

Target disease gene enrichment and sequencing

A total of 249 bone disease genes (Supplementary Table S1) were captured using a GenCap custom enrichment kit (MyGenostics, Beijing, China) based on previously described technologies. ${ }^{3,4}$ The capture experiment was conducted according to the manufacturer's protocol. In brief, genomic DNA from the proband was fragmented and then mixed with the GenCap probe (MyGenostics, Rockville, MD, USA) for PCR and hybridization. Then, MyOne beads (Life Technologies, Gaithersburg, MD, USA) were washed three times in $1 \times$ binding buffer and resuspended in $1 \times$ binding buffer. Subsequently, $2 \times$ binding buffer was added to the hybrid mix, which was then transferred to the tube with the MyOne beads. The mix was agitated on a rotator. The beads were then washed once with WB1 buffer at room temperature for $15 \mathrm{~min}$ and then three times with WB3 buffer at $65^{\circ} \mathrm{C}$ for $15 \mathrm{~min}$. The bound DNA was eluted with buffer elute. Finally, the eluted DNA was amplified for 15 cycles, and the PCR product was purified using SPRI (solid phase reversible immobilization) beads (Beckman Coulter, Miami, FL, USA) according to the manufacturer's protocol. The enriched libraries were sequenced using a Illumina HiSeq 2000 sequencer (Illumina, San Diego, CA, USA) using 100-base pair paired-end reads.

\section{Bioinformatics analysis, Sanger validation and co-segregation} analysis

After Illumina HiSeq 2000 sequencing, the low-quality reads and adaptor sequences were filtered out using the Solexa QA package (Massey University, Palmerston North, New Zealand) ${ }^{5}$ and the cutadapt program (http://code.google.com/p/cutadapt/), respectively. Subsequently, the SOAP aligner program ${ }^{6}$ was used to align the remaining high-quality sequencing reads to the reference human genome (hg19). Variant calls were made using the SOAPsnp program ${ }^{6}$ and, after the removal of PCR duplicates by the Picard software, ${ }^{7}$ were realigned to hg19 using the
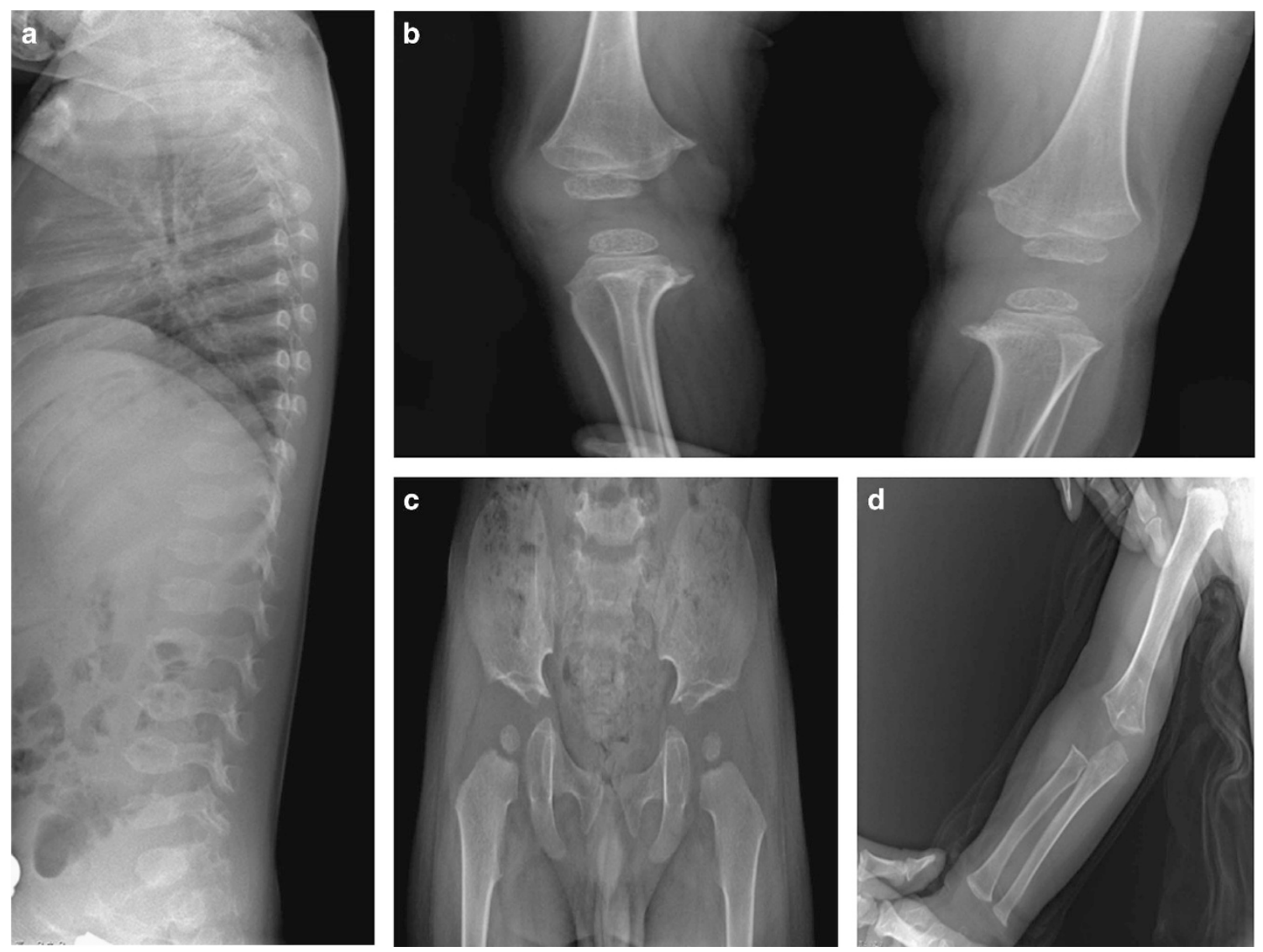

Figure 2. Radiographic findings in the PSACH proband. (a) Radiograph showing anterior beaking of the vertebral bodies. (b) Radiograph of knees. (c) Radiograph showing small femoral heads, flared metaphyseal borders and widened symphysis pubis. (d) Radiograph of ulna and radius. PSACH, pseudoachondroplasia. 
Burrows-Wheeler Aligner ${ }^{8}$ to identify insertions and deletions (InDels) using the genome analysis toolkit program. ${ }^{9}$ The identified single-nucleotide polymorphisms and InDels were annotated using the Exome-assistant program (http://122.228.158.106/exomeassistant). The MagicViewer software ${ }^{10}$ was used to view the short-read alignments and validate the candidate single-nucleotide polymorphisms and InDels. Finally, non-synonymous variants were evaluated using four algorithms, PolyPhen, SIFT, PANTHER and PMut as described previously, to predict the pathogenic potential of the variants. ${ }^{11}$

All suspected PSACH-causing mutations found by NGS were validated by direct Sanger sequencing. DNA sequences were obtained from the University of California Santa Cruz (UCSC) Genome Browser. Primer3.0 was used to design the primers (Supplementary Table S2). ${ }^{12}$ The amplicons were sequenced on an ABI 3730xl sequencer (Life Technologies). All family members were subsequently sequenced to perform co-segregation analysis.

\section{RESULTS}

Clinical information and bone radiographic findings

The proband (II-1) was 3 years old and $77 \mathrm{~cm}$ tall. He had strikingly short limbs (upper limb length $42 \mathrm{~cm}$ and lower limb length $33 \mathrm{~cm}$ ), small hands, short fingers and a waddling gait. A lateral spine radiograph (Figure 2a) showed anterior beaking of the vertebrae and platyspondyly. The pelvis radiograph (Figure 2c) showed small femoral heads, irregular acetabulae, enlarged acetabular angles and a widened symphysis pubis. Radiographs further indicated epiphyseal and metaphyseal changes in the joints of the long and short tubular bones including the femur, tibia, fibula, ulna and radius (Figure $2 \mathrm{~b}, \mathrm{~d}$ ). The radiographs and clinical features of the proband were reviewed by at least two clinical geneticists and radiologists, and the proband was diagnosed with suspected PSACH.

Targeted exome sequencing and co-segregation testing revealed candidate mutations

We performed targeted exome sequencing of 249 genes implicated in inherited bone disease. For the sample subjected to NGS, the average sequencing depth on the targeted regions was $103.28 \times$, and $98.0 \%$ of the targeted regions were covered. By using the SOAPsnp and genome analysis toolkit programs, ${ }^{6,9}$

Table 1. Number of candidate SNP/InDels filtered against several public variation databases and the in-house data

\begin{tabular}{lc}
\hline Filter & Feature_SNPS/InDels \\
\hline Total number of variants & 290 \\
Functional_SNP/InDels & 110 \\
Filtered_DBsnp/indel_1000 Genome (2012) & 21 \\
Filtered NIEHS Exome Sequencing database & 11 \\
(6500) & \\
Filtered in-house data & 6 \\
Filtered clinical phenotypes & 2
\end{tabular}

Abbreviations: NIEHS, National Institute of Environmental Health Sciences; SNP, single-nucleotide polymorphism
290 variants were identified (Table 1), among which were 110 non-synonymous variants, including both missense and splicing variants. After filtering for common polymorphisms with a frequency higher than $0.5 \%$ in any of the available databases, including the 1000 Genome (2012), National Institute of Environmental Health Sciences Exome Sequencing database and an our internal control database, the number of candidate variants was narrowed down to six (Supplementary Table S3). Combining the computational predictions of four algorithms (PolyPhen, SIFT, PANTHER and PMut) and any documented clinical phenotypes, the candidate mutations were reduced to two single-nucleotide polymorphisms (Supplementary Table S4). Using the stepwise variant-filtering strategy as described, two candidate mutations in the proband were successfully identified (Table 2).

To confirm the NGS results, we conducted Sanger sequencing to validate the two candidate mutations in this family. The novel variant, c.1160_1162del (p.C387del) within the COMP gene, was confirmed and was only found in the proband as a heterozygous allele; the two unaffected parents did not harbor this variant (Figure 3). However, the second heterozygous variant, c.6631G > A (p.V2211l) of the ACAN gene, did not co-segregate with the phenotype of PSACH in this pedigree, as it was detected in both the affected proband and the unaffected father (Figure 3). Moreover, by conservative analysis, p.C387 of COMP is highly conserved among mammals (Figure 4).

\section{DISCUSSION}

PSACH is an autosomal dominant osteochondrodysplasia that was first localized to human chromosome 19. ${ }^{1,13}$ Using genetic linkage analysis and mutation screening, the COMP gene at 19p13.1-p12 was identified as the gene responsible for the PSACH. ${ }^{14}$ The COMP gene is made up of 19 exons. Exons 4-19 encode the epidermal growth factor-like (type II) repeats, calmodulin-like (type III) repeats (CLRs) and the C-terminal domain, and exons 1-3 contain sequences unique to COMP. ${ }^{15}$ Over the last decade, considerable progress has been made in identifying the underlying genetic defects in PSACH. However, it now appears that PSACH results almost exclusively from mutations in the COMP gene. ${ }^{1}$ Through 2008, more than 60 COMP mutations were identified to be associated with PSACH, most of which ( 95\%) were clustered within exons $8-14$, within the CLR domains. The remaining $5 \%$ of the identified mutations were in exons 16 and 18, which encode the C-terminal domain. ${ }^{1,16,17}$ This underscores the importance of the CLRs for the structure of the COMP protein. Chen et al. ${ }^{18}$ proposed that type III CLRs have a role in binding calcium ions owing to the 13 calcium-binding loops that are consistent with the consensus sequence of an EF-hand calcium-binding loop.

In this study, the novel variant c.1160_1162del (p.C387del) in exon 11 of the COMP gene was identified, which is located in the fourth CLR (Figure 4). The mutation is in the 23-residue-long C-type motif, which is on the A chain of the crystal structure of the signature domain of COMP. ${ }^{19}$ There are nine disulfide bonds associated with the CLRs, including 6C-7C (C387-C407). ${ }^{19}$ Thus, the p.C387del mutation results in the disruption of this

Table 2. Candidate exome sequence variants filtered by database

\begin{tabular}{|c|c|c|c|c|c|c|c|c|}
\hline chr15:89402447 & $A C A N$ & Exon 12: c.6631G > A, p.V2211। & Heterozygous & $\begin{array}{l}\text { Non-synonymous, } \\
\text { SNV }\end{array}$ & Novel & 25 & $\begin{array}{l}\text { Tolerated, } \\
\text { NA }\end{array}$ & $N A, N A$ \\
\hline chr19:18897434-18897438 & COMP & $\begin{array}{l}\text { Exon 11: c.1160_1162del, } \\
\text { p.C387del }\end{array}$ & Heterozygous & $\begin{array}{l}\text { Nonframeshift, } \\
\text { deletion }\end{array}$ & Novel & 73 & $N A, N A$ & IAA, IAA \\
\hline
\end{tabular}



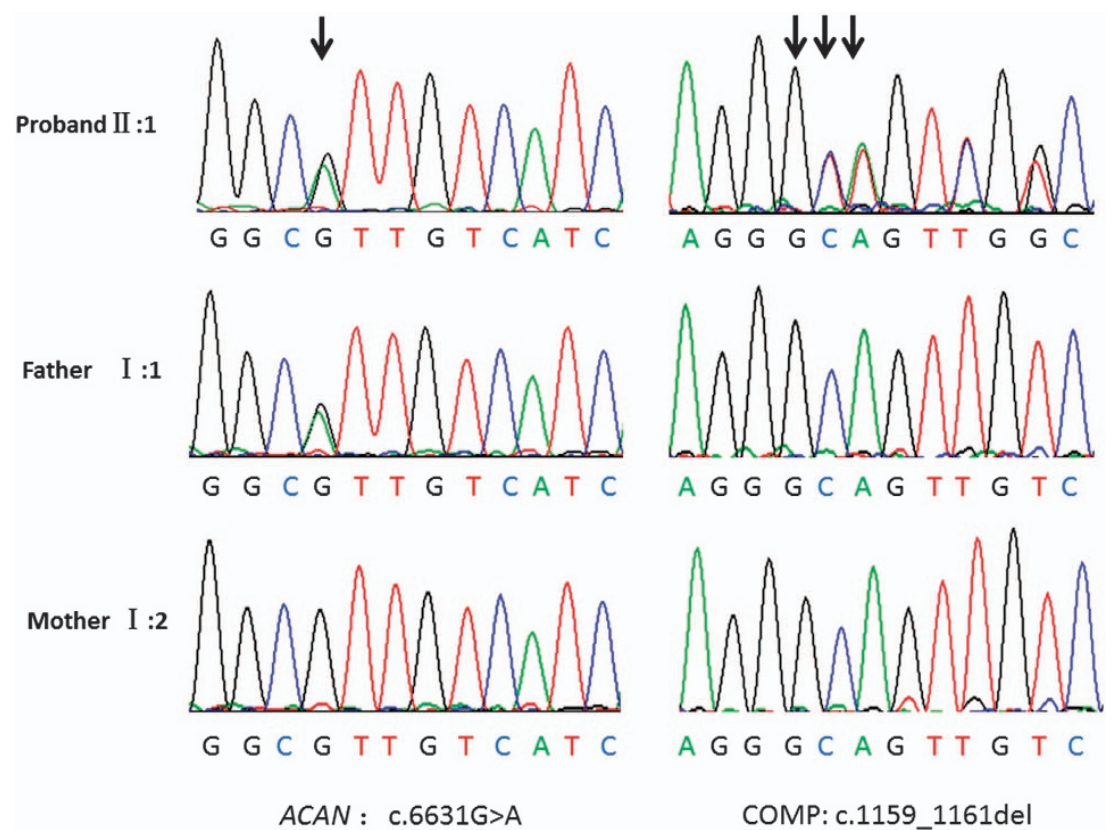

COMP: c.1159_1161del

Figure 3. Mutation identification of $A C A N$ gene and COMP gene. Electropherogram analysis of ACAN gene and COMP gene in suspected PSACH family showing the co-segregation of the heterozygous mutation c.1160_1162del of the COMP gene with the phenotype. II- 1 proband harbored heterozygous alleles, but neither the father I-1 nor the mother I- 2 carried this mutation. PSACH, pseudoachondroplasia.

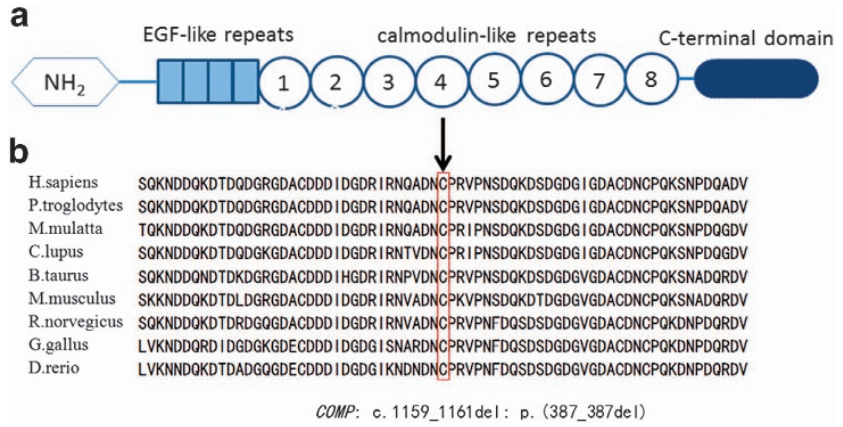

Figure 4. Structural domains of COMP and conservation analysis. (a) Structural domains of COMP and the location of c.1160_1162del are shown by the arrow. (b) Results obtained from HomoloGene showing that residue 387 in the COMP protein is conserved among different species.

disulfide bond. A missense variant, c. 1159T > G (p.C387G), at the same position, was reported by lkegawa et al. ${ }^{20}$ in a patient with mild PSACH. Moreover, this novel variant is located at a highly conserved position among several species (Figure 4), which suggests a strong functional and structural constraint.

As an extracellular matrix protein specific to cartilage, COMP catalyses the assembly of collagens and promotes formation of well-defined fibrils. ${ }^{21}$ Mutations in the CLR repeats are thought to affect the ability of the protein to bind $\mathrm{Ca}^{2+}$ and fold into the normal conformation. ${ }^{22}$ The mutant COMP and other extracellular matrix proteins are retained within the rough endoplasmic reticulum of chondrocytes with the help of chaperone proteins and are not degraded. ${ }^{22,23}$ Thus, the accumulation of mutant COMP may compromise chondrocyte function, ultimately leading to chondrocyte death 24,25 .

According to the results of the physical examination and radiographic evaluation, the proband was diagnosed with suspected PSACH. After the NGS sequence analysis and cosegregation testing, the COMP variant c.1160_1162del was identified as a novel mutation responsible for this mild familial form of PSACH. In this study, we conducted a NGS-based comprehensive molecular diagnosis of a Chinese PSACH patient. As a novel re-sequencing technology, NGS can detect mutations in many different suspect genes at a lower cost and in a time-efficient manner by generating deep coverage of the target sequences. With the improvement in gene enrichment, the accuracy of sequencing chemistries, computational algorithms for alignment and bioinformatic tools, all known and candidate bone disease genes can be tested simultaneously. Genetic screening of multiple genes involved in bone disease based on NGS technologies may therefore significantly benefit the comprehensive diagnosis of early-stage PSACH and, consequently, reproductive decision-making and genetic counseling for affected families.

\section{ACKNOWLEDGEMENTS}

We thank all family members for their participation. This work was supported by grants from the Natural Science Foundation of China (81170883 and 81430008 (ZY)) and the Department of Science and Technology of Sichuan Province (2014SZ0169, 2015 SZ0052 (ZY) and $2015 S Z 0060(\mathrm{FL}))$.

\section{COMPETING INTERESTS}

The authors declare no conflict of interest.

\section{REFERENCES}

1 Briggs MD, Chapman KL. Pseudoachondroplasia and multiple epiphyseal dysplasia: mutation review, molecular interactions, and genotype to phenotype correlations. Hum Mutat 2002; 19: 465-478.

2 Posey KL, Alcorn JL, Hecht JT. Pseudoachondroplasia/COMP — translating from the bench to the bedside. Matrix Biol 2014; 37: 167-173.

3 He J, Wu J, Jiao Y, Wagner-Johnston N, Ambinder RF, Diaz LA Jr et al. IgH gene rearrangements as plasma biomarkers in Non-Hodgkin's lymphoma patients. Oncotarget 2011; 2: 178-185.

4 Wu J, Matthaei H, Maitra A, Dal Molin M, Wood LD, Eshleman JR et al. Recurrent GNAS mutations define an unexpected pathway for pancreatic cyst development. Sci Transl Med 2011; 3: 92ra66.

5 Cox MP, Peterson DA, Biggs PJ. SolexaQA: at-a-glance quality assessment of Illumina second-generation sequencing data. BMC Bioinformatics 2010; 11: 485. 
6 Li R, Yu C, Li Y, Lam TW, Yiu SM, Kristiansen K et al. SOAP2: an improved ultrafast tool for short read alignment. Bioinformatics 2009; 25: 1966-1967.

7 Li H, Handsaker B, Wysoker A, Fennell T, Ruan J, Homer N et al. The Sequence Alignment/Map format and SAMtools. Bioinformatics 2009; 25: 2078-2079.

$8 \mathrm{Li} \mathrm{H}$, Durbin R. Fast and accurate short read alignment with Burrows-Wheeler transform. Bioinformatics 2009; 25: 1754-1760.

9 DePristo MA, Banks E, Poplin R, Garimella KV, Maguire JR, Hartl C et al. A framework for variation discovery and genotyping using next-generation DNA sequencing data. Nat Genet 2011; 43: 491-498.

10 Hou H, Zhao F, Zhou L, Zhu E, Teng H, Li X et al. MagicViewer: integrated solution for next-generation sequencing data visualization and genetic variation detection and annotation. Nucleic Acids Res 2010; 38: W732-W736.

11 Jin ZB, Mandai M, Yokota T, Higuchi K, Ohmori K, Ohtsuki F et al. Identifying pathogenic genetic background of simplex or multiplex retinitis pigmentosa patients: a large scale mutation screening study. J Med Genet 2008; 45: $465-472$.

12 Rozen S, Skaletsky H. Primer3 on the WWW for general users and for biologist programmers. Methods Mol Biol 2000; 132: 365-386.

13 Briggs MD, Rasmussen IM, Weber JL, Yuen J, Reinker K, Garber AP et al. Genetic linkage of mild pseudoachondroplasia (PSACH) to markers in the pericentromeric region of chromosome 19. Genomics 1993; 18: 656-660.

14 Hecht JT, Nelson LD, Crowder E, Wang Y, Elder FF, Harrison WR et al. Mutations in exon 17B of cartilage oligomeric matrix protein (COMP) cause pseudoachondroplasia. Nat Genet 1995; 10: 325-329.

15 Briggs MD, Hoffman SM, King LM, Olsen AS, Mohrenweiser H, Leroy JG et al. Pseudoachondroplasia and multiple epiphyseal dysplasia due to mutations in the cartilage oligomeric matrix protein gene. Nat Genet 1995; 10: 330-336.

16 Stenson PD, Mort M, Ball EV, Howells K, Phillips AD, Thomas NS et al. The Human Gene Mutation Database: 2008 update. Genome Med 2009; 1: 13.

17 Wang $\mathrm{CH}$, Lin WD, Tsai A, Tsai FJ. Novel human pathological mutations. Gene symbol: COMP. Disease: pseudoachondroplasia. Hum Genet 2009; 125: 350.
18 Chen H, Deere M, Hecht JT, Lawler J. Cartilage oligomeric matrix protein is a calcium-binding protein, and a mutation in its type 3 repeats causes conformational changes. J Biol Chem 2000; 275: 26538-26544.

19 Tan K, Duquette M, Joachimiak A, Lawler J. The crystal structure of the signature domain of cartilage oligomeric matrix protein: implications for collagen, glycosaminoglycan and integrin binding. FASEB J 2009; 23: 2490-2501.

20 Ikegawa S, Ohashi H, Nishimura G, Kim KC, Sannohe A, Kimizuka M et al. Novel and recurrent COMP (cartilage oligomeric matrix protein) mutations in pseudoachondroplasia and multiple epiphyseal dysplasia. Hum Genet 1998; 103: 633-638.

21 Halasz K, Kassner A, Morgelin M, Heinegard D. COMP acts as a catalyst in collagen fibrillogenesis. J Biol Chem 2007; 282: 31166-31173.

22 Unger S, Hecht JT. Pseudoachondroplasia and multiple epiphyseal dysplasia: New etiologic developments. Am J Med Genet 2001; 106: 244-250.

23 Hecht JT, Hayes E, Snuggs M, Decker G, Montufar-Solis D, Doege K et al. Calreticulin, PDI, Grp94 and BiP chaperone proteins are associated with retained COMP in pseudoachondroplasia chondrocytes. Matrix Biol 2001; 20: 251-262.

24 Duke J, Montufar-Solis D, Underwood S, Lalani Z, Hecht JT. Apoptosis staining in cultured pseudoachondroplasia chondrocytes. Apoptosis 2003; 8: 191-197.

25 Hecht JT, Montufar-Solis D, Decker G, Lawler J, Daniels K, Duke PJ. Retention of cartilage oligomeric matrix protein (COMP) and cell death in redifferentiated pseudoachondroplasia chondrocytes. Matrix Biol 1998; 17: 625-633.

c) (i) $\Theta$ This work is licensed under a Creative Commons AttributionNonCommercial-NoDerivs 4.0 International License. The images or other third party material in this article are included in the article's Creative Commons license, unless indicated otherwise in the credit line; if the material is not included under the Creative Commons license, users will need to obtain permission from the license holder to reproduce the material. To view a copy of this license, visit http:// creativecommons.org/licenses/by-nc-nd/4.0/

Supplementary Information for this article can be found on the Human Genome Variation website (http://www.nature.com/hgv) 